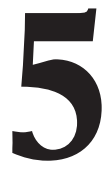

\title{
VALORACIÓN DE LA PRÁCTICA ORIENTADORA Y TUTORIAL EN LA UNIVERSIDAD: VALIDACIÓN DE UNA ESCALA PARA EL ALUMNADO
}

\author{
(ASSESSMENT OF THE GUIDING AND TUTORIAL PRACTICE AT \\ UNIVERSITY: VALIDATION OF A SCALE FOR THE STUDENTS)
}

Antonio Pantoja-Vallejo

David Molero

Universidad de Jaén

M. Dolores Molina-Jaén

Centro de Profesorado Universitario SAFA Úbeda

Adscrito a la Universidad de Jaén

M. Jesús Colmenero-Ruiz

Universidad de Jaén

DOI: $10.5944 / e d u c X X 1.25632$

\section{Cómo referenciar este artículo/How to reference this article:}

Pantoja-Vallejo, A.; Molero, D.; Molina-Jaén, M.D. y Colmenero-Ruiz, M.J. (2020). Valoración de la práctica orientadora y tutorial en la universidad: validación de una escala para el alumnado. Educación XX1, 23(2), 119-143, doi: 10.5944/educXX1.25632

Pantoja-Vallejo, A.; Molero, D.; Molina-Jaén, M.D. \& Colmenero-Ruiz, M.J. (2020). Assessment of the guiding and tutorial practice at university: validation of a scale for the students. Educación XX1, 23(2), 119-143, doi: 10.5944/educXX1.25632

\section{RESUMEN}

La labor tutorial del profesorado universitario está ligada al fomento de acciones orientadoras, de muy reciente implantación en los estudios superiores, independientemente de las materias o áreas de las que se trate. Esto ha conllevado una falta de formación específica del profesorado universitario para atender demandas del alumnado. El objetivo de este trabajo fue validar la escala denominada POTAE-17, cuya finalidad es la de medir y valorar la práctica orientadora y tutorial en el alumnado y egresados universitarios. La muestra representativa, siguiendo un sistema de muestreo aleatorio proporcional, estuvo compuesta por 4003 estudiantes matriculados en $2^{\circ}$ y $4^{\circ}$ curso de las 24 titulaciones comunes en las universidades españolas de Granada y Jaén, máster y doctorado (35.22\% hombres; 
$64.77 \%$ mujeres). A la misma se suman las otras instituciones participantes en el estudio general, Instituto Politécnico de Coímbra (Portugal) y Queen Mary University of London (Reino Unido), si bien los sujetos que aportan no presentan proporcionalidad al no haber coincidencia de estudios en estos países. Para el análisis de los datos se utilizó el paquete R Project for Statistical Computing y Lavaan de R. La escala POTAE-17 reflejó una estructura de 4 factores (orientación académica, orientación personal, orientación profesional, y orientación y Tecnologías de la Información y la Comunicación), mostrando la multidimensionalidad de la escala y una adecuada fiabilidad y validez. Estos resultados se apoyan en la necesidad de disponer de una medida que sea útil para el profesorado y alumnado en el ámbito de la orientación y la tutoría en el contexto universitario y, por ende, de fomentar la formación en acciones orientadoras universitarias.

\section{PALABRAS CLAVE}

Educación Superior; orientación; tutoría; propiedades psicométricas; validez; fiabilidad.

\section{ABSTRACT}

The tutorial work of university lecturers is linked to the promotion of orientating actions, very recently implemented in higher education, regardless of the subjects in question. This has led to a lack of specific training of university lecturers in order to meet the demands of the students. The aim of this study was to validate the scale called POTAE-17, the purpose of which is to measure and assess the guiding and tutorial practice in university students and graduates. The representative sample consisted of 4,003 students enrolled in the 2nd and 4th year of the 24 common degrees in the Spanish universities of Granada and Jaén, master's and doctorate (35.22\% men, $64.77 \%$ women). The sample also includes other institutions participating in the general study, such as the Polytechnic Institute of Coimbra (Portugal) and the Queen Mary University of London (United Kingdom), although the subjects who contribute do not show proportionality as there is no coincidence of studies in these countries. In order to analyse the data, the R Project for Statistical Computing and Lavaan de R. tools were used. The POTAE-17 scale reflected a structure which consisted of 4 factors (academic orientation, personal orientation, professional orientation, and orientation and ICT), showing the multiple dimensions of the scale and an adequate reliability and validity. These results are based on the need to have a measure that is useful for teachers and students in the field of guidance and tutoring in the university context. 


\section{KEYWORDS}

Higher Education; guidance; tutoring; psychometric properties; validity; reliability.

\section{INTRODUCCIÓN}

La labor tutorial del profesorado universitario, bien sea en las materias que imparte o en los Planes de Acción Tutorial (PAT), conlleva el fomento de acciones orientadoras (European Lifelong Guidance Policy Network, 2015) de muy reciente implantación en los estudios superiores. Esto ha supuesto una falta de formación específica del profesorado para atender demandas del alumnado, una escasa experiencia acumulada en los PAT y la necesidad de programas específicos y de materiales de ayuda, que deben implementarse en diversas áreas del desarrollo personal del estudiante universitario, atendiendo a las dimensiones intelectual cognitiva, afectivoemotiva, social y profesional (Rodríguez, 2004).

Para alcanzar esas metas, la tutoría se da en tres niveles de intervención, como son la tutoría de materia, de carrera y de asesoramiento personal. $\mathrm{Al}$ articular esta intervención hay que tener muy presente la interrelación de diferentes factores relacionados con la institución a la que pertenece el alumnado, la titulación y las características de los propios estudiantes, pero siempre, las acciones deben dirigirse a mejorar el rendimiento académico del alumnado (León y Lugo, 2008), ya que ayuda a integrarse en los estudios iniciados (Coertjens, Donche, De Maeyer, Van Daal, \& Van Petegem, 2017; Feldt, Graham, \& Dew, 2011), solucionar los problemas académico y/o escolares, y a que el alumnado desarrolle hábitos de estudio, de trabajo, de reflexión y de convivencia social, y además se ayuda a prevenir, con estas actuaciones, la posibilidad de que el alumnado abandone prematuramente sus estudios universitarios (Cambours, Iglesias, y Muiños, 2017), y/o que los oriente hacia un lado u otro, para lograr mejores resultados una vez que pueda acceder al mundo laboral, gestionando adecuadamente la transición (Holliman, Martin, \& Collie, 2018; Magen \& Shachar, 2017) .

Pero dando un paso más allá, es necesario un modelo de tutoría que se podría definir como integral (Castellano y Pantoja, 2017) y que, sin olvidar la atención al alumnado a la que hemos hecho referencia, debe posibilitar el desarrollo de competencias personales y profesionales, el logro de una acción multidisciplinar e interdisciplinar, un tiempo y espacio para la tutoría, el apoyo de la política institucional, la capacitación del profesorado y la incorporación de las TIC. 
La relevancia de la función tutorial ha hecho que la investigación sea amplia en esta temática, tanto a la hora de analizar las funciones que la definen (Álvarez, 2013; Campos y Oliveira, 2013), en la modalidad en la que se desarrolla (Cano, 2009; Capelari, 2014), o en las aportaciones que ofrece al alumnado (Larose, et al., 2011; Lorenzo, Carreres, Lledó, y Vera, 2017), en los instrumentos utilizados para determinar su calidad (Caldera, Carranza, Jiménez, y Pérez, 2015), o en su utilidad desde el punto de vista del alumnado (Álvarez Pérez, 2013; De la Cruz, 2017) o del profesorado, y en el uso de las TIC para su desarrollo (Pantoja, 2009). No obstante, el uso generalizado de las TIC en la sociedad actual, y la diversidad de herramientas que están a nuestro alcance, para el acceso a la información, ha hecho posible que los estudiantes empleen para la tutoría, aquellas que como demuestran Michavila y García (2003) y Rodríguez y Calvo (2011), dan como resultado la búsqueda de recursos.

Estudios recientes (Fernández, Mena, y Tójar, 2017) muestran las posibilidades de la tutoría como facilitadora del e-learning y cómo esta es la modalidad más utilizada en la formación continua, debido a las ventajas que presenta (Fernández y Cabero, 2016).

Las necesidades del alumnado universitario (Sánchez, López y Cienfuegos, 2018) se encuadran principalmente en tres aspectos como son el académico, el personal y el profesional. La orientación académica está orientada a la superación de la asignatura, demostrando las investigaciones la necesidad de este tipo de tutoría (Araiz, Berbegal, y Sabiron, 2018; Respondek, Seufert, Stupnisky, \& Nett, 2017). El apoyo personal (Wisker, Exley, Antoniou, \& Ridley, 2008) que desde la tutoría se ofrece al alumnado, hace que los resultados académicos sean mayores y, en parte, es debido a la relación cercana y horizontal que se establece en esta relación, sobre todo en los principios de sus estudios (Clerici \& Da Re, 2019; Tinto, 2017) posibilitando que se mantenga en la institución escolar más tiempo.

Según García Antelo (2011), la dimensión personal es la que más polémica ha despertado, en opinión del profesorado, por la falta de formación que posee para abordar los aspectos relacionados con ella y porque tradicionalmente la tutoría universitaria ha focalizado principalmente su atención en dar respuesta a las demandas académicas. No obstante, son numerosas las investigaciones (Amor y Dios, 2017; Muñoz y Gairin, 2016) que han demostrado que el desarrollo personal proporciona el logro de competencias académicas y sociales que facilitan el aumento en el rendimiento y en las posibilidades de inserción laboral. Son muchos los estudios que avalan que una relación personal desde la tutoría hace que el alumnado de educación superior permanezca en la institución (FarrWharton, Charles, Keast, Woolcott, \& Chamberlain, 2018; Van Rooij, Jansen, 
\& Van de Grift, 2017), ya que, como vemos en otros estudios recientes (Holliman, Martin, \& Collie, 2018), la relación con el centro es menor en la universidad que en otras etapas educativas.

En cuanto a la orientación profesional, la consideramos como un proceso de ayuda al estudiante, destinado a conseguir una comprensión adecuada de las distintas opciones profesionales (Watts, 1990) que existen en el mundo laboral, eligiendo aquella que cumpla con sus intereses y objetivos personales.

En resumen, el objetivo del presente estudio ha sido diseñar y validar una escala de conocimientos acerca de la tutoría universitaria en sus dimensiones, académica, personal, profesional y Tecnologías de la Información y la Comunicación (TIC) para ser cumplimentado por el alumnado, ya que consideramos importante "repensar la forma como se gestiona la tutoría universitaria en la enseñanza superior" (Venegas y Gairín, 2018, p. 125).

\section{MÉTODO}

\section{Participantes}

La población objeto de estudio la componen el total de estudiantes de la Universidad de Jaén (UJA) y de la Universidad de Granada (UGR) matriculados en $2^{\circ}$ y $4^{\circ}$ de las 24 titulaciones de grado comunes en ambas universidades, y postgrado de todo el conjunto de estudios universitarios, divididos en hombres y mujeres. La determinación de estudiantes de $2^{\circ}, 4^{\circ}$, máster o doctorado obedece al criterio de comprobar en qué medida afecta, en su caso, la orientación recibida durante el periodo universitario. De la misma, se ha extraído la muestra correspondiente, mediante un sistema de muestreo aleatorio estratificado y proporcional, con un nivel de confianza del $95 \%$ y un error muestral del $5 \%$. A esta muestra se suman las otras instituciones participantes en el estudio general, Instituto Politécnico de Coímbra (Portugal) y Queen Mary University of London (Reino Unido), si bien los participantes de estas universidades no han sido seleccionados de manera proporcional, al no haber coincidencia en la denominación de los estudios en estos países. En la tabla 1 se detalla la distribución de los participantes en función de los objetivos definidos para el estudio. La muestra tomada en consideración para la validación del instrumento está compuesta por 4003 estudiantes. 
Tabla 1

Muestra del estudio en sus principales variables

\begin{tabular}{rrrrrrr}
\hline & $\mathbf{2}^{\mathbf{o}}$ & $\mathbf{4}^{\mathbf{0}}$ & Máster & Doctorado & Hombre & Mujer \\
\hline $\mathrm{f}$ & 1875 & 1305 & 744 & 79 & 1410 & 2593 \\
$\%$ & 46.84 & 32.60 & 18.59 & 1.97 & 35.22 & 64.78 \\
\hline
\end{tabular}

\section{Instrumento de recogida de información}

Se construyó y validó ad hoc la escala "La práctica orientadora y tutorial en el alumnado y egresados universitarios (POTAE-17)", un instrumento de evaluación de las diferentes acciones que en orientación desarrolla el alumnado a lo largo de su vida universitaria y al término de la misma, cuando de alguna manera sigue ligado a la institución. Como se verá más adelante, analiza cuatro grandes dimensiones: orientación académica, orientación personal, orientación profesional y orientación y TIC. La fiabilidad de las puntuaciones (Alfa de Cronbach, consistencia interna) obtenidas en cada una de las dimensiones del instrumento, en la aplicación de la prueba piloto ha sido .89 para Orientación Académica, .85 para Orientación Personal, .94 para Orientación Profesional y .87 para Orientación y TIC; siendo la fiabilidad total de las puntuaciones del cuestionario de .96 .

\section{Procedimiento}

En función de los objetivos principales que se definieron para la investigación, tras una profunda búsqueda bibliográfica y una serie de reuniones del equipo investigador, se realizaron varias versiones de la escala y se llevaron a cabo las estrategias de definición de su validez y fiabilidad hasta llegar a los valores de calidad que permitieron configurar el instrumento definitivo. En su construcción participó, como se verá a continuación, profesorado de diferentes universidades públicas de España, Portugal y Reino Unido, y se garantizó en todo momento la confidencialidad de los datos. Para el análisis de los datos se utilizó el paquete R Project for Statistical Computing y Lavaan de R.

\section{RESULTADOS}

\section{Validez del instrumento}

Morales, Urosa, y Blanco (2000) señalan diversos pasos en la construcción de una escala, que se concretan en cuatro, una vez se tiene clara la actitud o 
rasgo que se desea medir: preparación del instrumento, obtención de datos de una muestra, análisis del instrumento y análisis adicionales. Tomando estas fases como referencia, el equipo investigador estuvo formado por un total de 14 profesores de universidad: UJA (4), UGR (5), Coimbra (3) y Londres (2). En primer lugar, valoran, tras una amplia revisión de la literatura y de diversos instrumentos de similares características, la agrupación de los ítems en torno a cuatro grandes dimensiones: orientación académica, personal, profesional y orientación y TIC. En las diferentes sesiones de trabajo se definen los distintos rasgos que se desean medir en cada dimensión, hasta que queda configurada una primera versión del instrumento. De forma paralela, se toma la decisión de evaluar la pertinencia de los ítems en relación con una serie de criterios considerados importantes para garantizar la calidad psicométrica de los mismos y, por tanto, validar la hipótesis de que representan adecuadamente el constructo. Estos indicadores son:

- Claridad: el ítem se comprende fácilmente.

- Coherencia: el ítem tiene relación lógica con la dimensión que está midiendo.

- Relevancia: es esencial la inclusión del ítem.

Con estas características, se diseña la primera versión de la escala "La práctica orientadora y tutorial en el alumnado y egresados universitarios (POTAE-17)", que cuenta con un total de 73 ítems, repartidos conforme se aprecia en la Tabla 2. Todos ellos incorporan un apartado para que cada juez pueda realizar observaciones en el sentido de añadir, cambiar o eliminar cualquier ítem o expresión que considere oportuno. Seguidamente, se eligen 10 jueces al azar ( 5 hombres y 5 mujeres), buscando representación en las cuatro universidades participantes y una experiencia como tutores entre 5 y más de 15 años. Valoran el grado de pertinencia de cada ítem en los tres indicadores en una escala de cuatro opciones de respuesta ( 1 =deficiente; $4=$ excelente). Una vez recogidas las respuestas, se reúne de nuevo el equipo investigador para realizar una valoración de las mismas, comprobándose frecuencias, porcentajes, medias y desviación típica de la concordancia con el constructo y con la pertinencia del ítem a la dimensión evaluada. Se eliminan aquellos ítems que no obtienen al menos un $75 \%$ de unanimidad y que corresponden con medias cercanas o por debajo de 2 en los tres indicadores. Seguidamente, se realiza la prueba $W$ de Kendall, con la finalidad de conocer el grado de asociación entre k conjuntos de rangos (Siegel y Castellan, 1995); en este caso, la concordancia entre las respuestas de los jueces, valorando las respuestas sin eliminar y eliminando los ítems mencionados anteriormente. De forma global el coeficiente $W$ es igual a .343 y no es significativo $\chi^{2}(215)=224.155 p \leq .373$, lo que indica que 
hay un adecuado índice de concordancia entre los jueces. Tras el filtrado correspondiente, la escala definitiva queda formada por 61 ítems.

Una vez modificados o excluidos los ítems, tras los análisis estadísticos, se realizó una revisión semántica, tomando como referencia las indicaciones emanadas de una mayoría de jueces. Se unificaron varios de los enunciados y se mejoró la redacción y comprensión de algunos ítems, buscando un lenguaje más apropiado a los estudiantes universitarios. De igual forma, se definieron 5 opciones de respuesta $(1=$ totalmente en desacuerdo; $5=$ totalmente de acuerdo).

Tabla 2

Versiones inicial y final de la escala

\begin{tabular}{lcc}
\hline \multicolumn{1}{c}{ Dimensión } & Ítems versión inicial & Ítems versión final \\
\hline Orientación académica & 25 & 18 \\
Orientación personal & 15 & 13 \\
Orientación profesional & 17 & 16 \\
Orientación y TIC & 16 & 14 \\
\hline \multicolumn{1}{c}{ Totales } & $\mathbf{7 3}$ & $\mathbf{6 1}$ \\
\hline
\end{tabular}

A continuación, se analizan las evidencias de validez de constructo de los ítems del instrumento, mediante un análisis factorial exploratorio. Se ha utilizado la matriz de correlación policórica al tratarse de variables ordinales con cinco categorías; es decir, solo se utilizan en la validación estos ítems y no la variable final de cada escala que se puntúa de 1 a 10. El propósito ha sido establecer los componentes principales del cuestionario y determinar la varianza que estos explican y ver si existía una concordancia con los factores propuestos por los revisores en la validación del contenido del mismo.

El grado de significación de los coeficientes, obtenido en la matriz de correlaciones entre variables, en un contraste unilateral, resulta significativo en todos los casos $p<.000$. La medida de adecuación muestral de KaiserMeyer-Olkin (KMO) es de un valor próximo a la unidad, siendo por tanto el análisis factorial un procedimiento adecuado. El valor de KMO en nuestro estudio análisis es de .853 y se puede considerar como que procede la realización del análisis factorial.

Tabla 3

Valor de la prueba KMO y prueba de esfericidad de Bartlett

\begin{tabular}{lc}
\hline Medida de adecuación muestral del Kaiser-Meyer-Olkin & .853 \\
Prueba de esfericidad de Bartlett - Chi cuadrado & 6701.698
\end{tabular}


Al utilizar el test de Bartlett verificamos si la matriz de correlaciones es una matriz de identidad; es decir, si todos los coeficientes de la diagonal son iguales a la unidad y los extremos de la diagonal iguales a 0 . A través de las transformaciones Chi cuadrado del determinante de la matriz de correlaciones comprobamos que cuanto mayor sea su valor, y por tanto, menor su grado de significación, es más improbable que la matriz sea una matriz de identidad. Para nuestro instrumento, Chi cuadrado tiene un valor de 6701.698 y una significación $p=.000$, por lo que queda de manifiesto que no es una matriz de identidad. Esto nos lleva a afirmar que el análisis factorial resulta pertinente.

De igual forma, se halló la medida de adecuación muestral (MSA) con la finalidad de comparar las magnitudes de los coeficientes de correlación observados y las correspondientes a la correlación parcial (Gil, 2003). Se obtiene un índice de .97, que se puede considerar muy alto, al igual que los distintos valores entre ítems que se aprecian en la Tabla 4, que además se mantienen muy estables.

Tabla 4

MSA item a item

\begin{tabular}{lllllllllll}
\hline Ítem & OA1 & OA2 & OA3 & OA4 & OA5 & OA6 & OA7 & OA8 & OA9 & OA10 \\
MSA & .96 & .96 & .97 & .98 & .98 & .98 & .98 & .97 & .98 & .98 \\
Ítem & OA11 & OA12 & OA13 & OA14 & OA15 & OA16 & OA17 & OA18 & OPER1 & OPER2 \\
MSA & .98 & .71 & .74 & .97 & .98 & .96 & .96 & .96 & .95 & .97 \\
Ítem & OP3 & OP4 & OP5 & OP6 & OP7 & OP8 & OP9 & OP10 & OP11 & OP12 \\
MSA & .98 & .96 & .96 & .98 & .94 & .92 & .96 & .98 & .98 & .97 \\
Ítem & OP13 & OPR1 & OPR2 & OPR3 & OPR4 & OPR5 & OPR6 & OPR7 & OP78 & OPR9 \\
MSA & .07 & .98 & .97 & .97 & .97 & .98 & .97 & .98 & .96 & .97 \\
Ítem & OPR10 & OPR11 & OPR12 & OPR13 & OPR14 & OPR15 & OPR16 & OT1 & OT2 & OT3 \\
MSA & .98 & .97 & .98 & .95 & .96 & .97 & .98 & .97 & .94 & .91 \\
Ítem & OT4 & OT5 & OT6 & OT7 & OT8 & OT9 & OT10 & OT11 & OT12 & OT13 \\
MSA & .91 & .93 & .92 & .95 & .96 & .94 & .95 & .95 & .96 & .95 \\
Ítem & OT14 & & & & & & & & & \\
MSA & .98 & & & & & & & & & \\
\hline
\end{tabular}

Nota: OA (Orientación Académica); OP (Orientación Personal); OPR (Orientación Profesional); OT (Orientación y TIC) 
El modelo de extracción de factores utilizado es el de Componentes Principales (MCP), ya que es, posiblemente, la técnica más empleada y aceptada en la investigación socioeducativa (Gaviria, 2000). Para llevarlo a cabo se realiza un análisis paralelo para determinar el número de factores, al comparar los autovalores empíricos con los aleatorios. Este tipo de análisis es válido para variables categóricas -tal es el caso del cuestionario POTAE- como señalan Liu y Rijmen (2008), Weng y Cheng (2005). La figura 1 muestra la existencia clara de tres factores y un cuarto que también permite considerarse por proximidad. Por su parte, el análisis factorial correspondiente, explica un 50\% de la varianza (Tabla 5).

\section{Parallel Analysis}

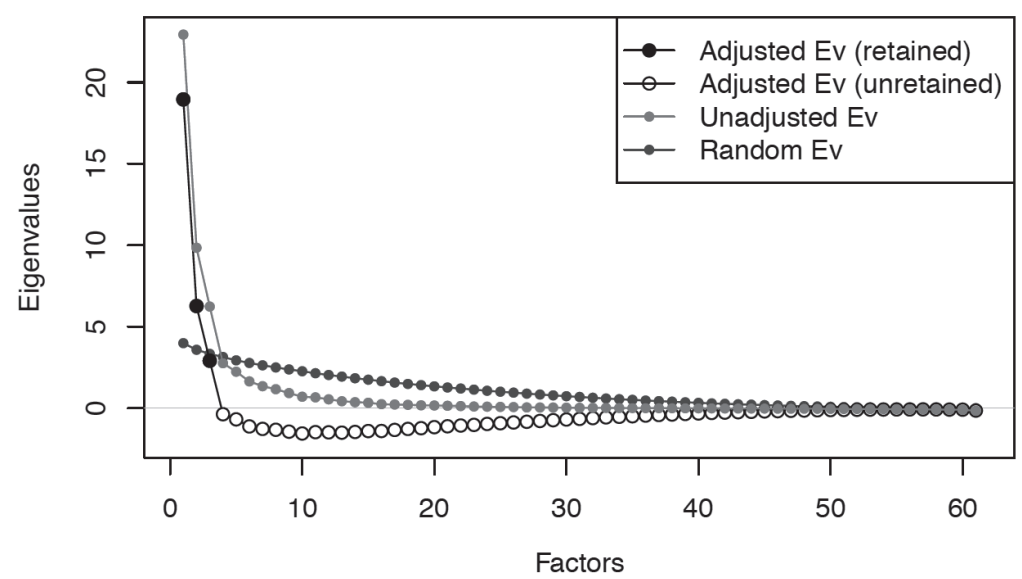

Figura 1. Análisis paralelo y factores extraídos

Tabla 5

Análisis de varianza

\begin{tabular}{lcccc}
\hline & RC1 & RC2 & RC3 & RC4 \\
\hline SS loading & 11.77 & 9.63 & 5.46 & 3.42 \\
\hline Proportion Var & .19 & .16 & .09 & .06 \\
\hline Cumulative Var & .19 & .35 & .44 & .50 \\
\hline Proportion Explained & .39 & .32 & .18 & .11 \\
\hline Cumulative Proportion & .39 & .71 & .89 & 1.00 \\
\hline
\end{tabular}

Mean item complexity $=1.8$

Test of the hypothesis that 4 compoments are sufficient

The root mean square of the residuals (RMSR) is .05

Fit based upon off diagonal values $=.98$ 
A continuación, se determina la estructura factorial de la escala, mediante un análisis factorial exploratorio, para lo cual se emplea el método de extracción con rotación Varimax, quedando agrupados los ítems tal y como se aprecia en la Tabla 6. Los cuatro factores identificados corresponden, como se ha expuesto con anterioridad, a Orientación Académica (OA); Orientación Personal (OP); Orientación Profesional (OPR) y Orientación y TIC (OT).

Tabla 6

Estructura factorial de la escala (rotación Varimax)

\begin{tabular}{|c|c|c|c|c|c|}
\hline \multirow{2}{*}{ Factor } & \multirow{2}{*}{ Ítem } & \multicolumn{4}{|c|}{ Cargas de los factores } \\
\hline & & 1 & 2 & 3 & 4 \\
\hline & OA9 & & .70 & & \\
\hline & OA6 & & .67 & & \\
\hline & OA2 & & .64 & & \\
\hline & OA1 & & .61 & & \\
\hline & OA 5 & & .61 & & \\
\hline & OA10 & & .60 & & \\
\hline $\mathrm{OA}$ & OA4 & & .59 & & \\
\hline & OA18 & & .57 & & \\
\hline & OA11 & & .54 & & \\
\hline & OA3 & & .53 & & \\
\hline & OA 7 & & .53 & & \\
\hline & OA17 & & .53 & & \\
\hline & OA8 & & .46 & & \\
\hline & OA12 & & .46 & & \\
\hline & OA16 & & .42 & & \\
\hline & OA15 & & .39 & & \\
\hline & OA14 & & .37 & & \\
\hline & OA 13 & & .11 & & \\
\hline & OP23 & & & & .63 \\
\hline & OP28 & & & & .58 \\
\hline & OP24 & & & & .54 \\
\hline & OP20 & & & & .52 \\
\hline OP & OP22 & & & & .52 \\
\hline & OP31 & & & & .50 \\
\hline & OP21 & & & & .49 \\
\hline & OP30 & & & & .46 \\
\hline & OP19 & & & & .45 \\
\hline & OP26 & & & & .45 \\
\hline & OP29 & & & & .44 \\
\hline
\end{tabular}




\begin{tabular}{|c|c|c|c|c|c|}
\hline \multirow{12}{*}{ Factor } & \multirow{2}{*}{ Ítem } & \multicolumn{4}{|c|}{ Cargas de los factores } \\
\hline & & 1 & 2 & 3 & 4 \\
\hline & OP25 & & & & .40 \\
\hline & OP27 & & & & .28 \\
\hline & OPR40 & .80 & & & \\
\hline & OPR38 & .79 & & & \\
\hline & OPR36 & .77 & & & \\
\hline & OPR41 & .77 & & & \\
\hline & OPR35 & .75 & & & \\
\hline & OPR39 & .75 & & & \\
\hline & OPR34 & .73 & & & \\
\hline & OPR37 & .71 & & & \\
\hline \multirow[t]{14}{*}{ OPR } & OPR33 & .69 & & & \\
\hline & OPR42 & .69 & & & \\
\hline & OPR32 & .65 & & & \\
\hline & OPR43 & .64 & & & \\
\hline & OPR47 & .64 & & & \\
\hline & OPR46 & .58 & & & \\
\hline & OPR45 & .52 & & & \\
\hline & OPR44 & .47 & & & \\
\hline & OT52 & & & .68 & \\
\hline & OT58 & & & .68 & \\
\hline & OT60 & & & .68 & \\
\hline & OT59 & & & .63 & \\
\hline & OT49 & & & .60 & \\
\hline & OT53 & & & .59 & \\
\hline \multirow[t]{8}{*}{ OT } & OT50 & & & .55 & \\
\hline & OT51 & & & .55 & \\
\hline & OT48 & & & .51 & \\
\hline & OT57 & & & .51 & \\
\hline & OT61 & & & .26 & \\
\hline & OT55 & & & .17 & \\
\hline & OT56 & & & .16 & \\
\hline & OT54 & & & .15 & \\
\hline
\end{tabular}

Aun cuando algunos valores de los factores son reducidos, se han dejado en blanco las cargas que son inferiores al valor del factor. Por otra parte, el análisis de fiabilidad de las escalas y el análisis factorial confirmatorio han corroborado la estructura factorial que muestra la Tabla 6. 
El último paso en el proceso de validación ha sido el análisis factorial confirmatorio (AFC) mediante la técnica SEM (Structural Equation Modeling), que permite representar el efecto causal entre las variables existentes en cualquier modelo teórico formulado, como continuación de técnicas multivariadas como el análisis factorial (Byrne, 2006), ya realizado anteriormente. Para ello se ha utilizado el paquete estadístico Lavaan de R. Como una medida de ajuste general de modelo se calcula el estadístico chicuadrado $\left(\chi^{2}\right)$ que, al ser significativo $\left(\chi^{2}=31984.829 ; p=.000\right)$, solo nos sirve para graduar la aproximación entre distintos modelos de ajuste. Por tanto, se necesita la confluencia de otros índices para dirimir si el ajuste del modelo a los datos es apropiado. Uno de los más apropiados es el RMSEA (Error Medio Cuadrático de Aproximación) al estar modulado por los grados de libertad de la muestra. No obstante, también se han utilizado otros índices de ajuste, que se muestran en la Tabla 7. En la misma, se aprecia cómo algunos indicadores están fuera del valor estándar, pero muy próximos al mismo, por los que se asumen como adecuados. En el caso del RMSEA presenta un buen ajuste de acuerdo a Steiger y Lind (1980).

Tabla 7

Medidas de ajuste del modelo

\section{Medidas de ajuste}

Índice de bondad de ajuste comparativo (CFI)

Índice de Tucker-Lewis (TLI)

Índice de bondad de ajuste (GFI)

Índice de bondad de ajuste corregido (AGFI)

Raíz del residuo cuadrático promedio (RMR)

Raíz del residuo cuadrático promedio de aproximación (RMSEA)

$\begin{array}{ll}\begin{array}{l}\text { Valor } \\ \text { escala }\end{array} & \begin{array}{l}\text { Valor } \\ \text { estándar }\end{array} \\ .743 & \geq .95 \\ .734 & \geq .95 \\ .701 & \geq .95 \\ .853 & \geq .95 \\ .067 & \text { Próximo a } 0 \\ .066 & <.08\end{array}$

Las relaciones estructurales observadas en las diferentes variables analizadas, se muestran representadas en la figura 2. Los valores existentes en las flechas unidireccionales señalan la influencia que existe entre las variables latentes representadas con una elipse (factores) y los valores de la escala. En el centro se muestran los pesos que cada uno de los ítems tienen sobre el factor y en la parte izquierda se representan los errores de medida (error aleatorio + la varianza sistemática debida a otros elementos que el indicador mide, además del factor subyacente, tales como los efectos de un particular método de medida). 


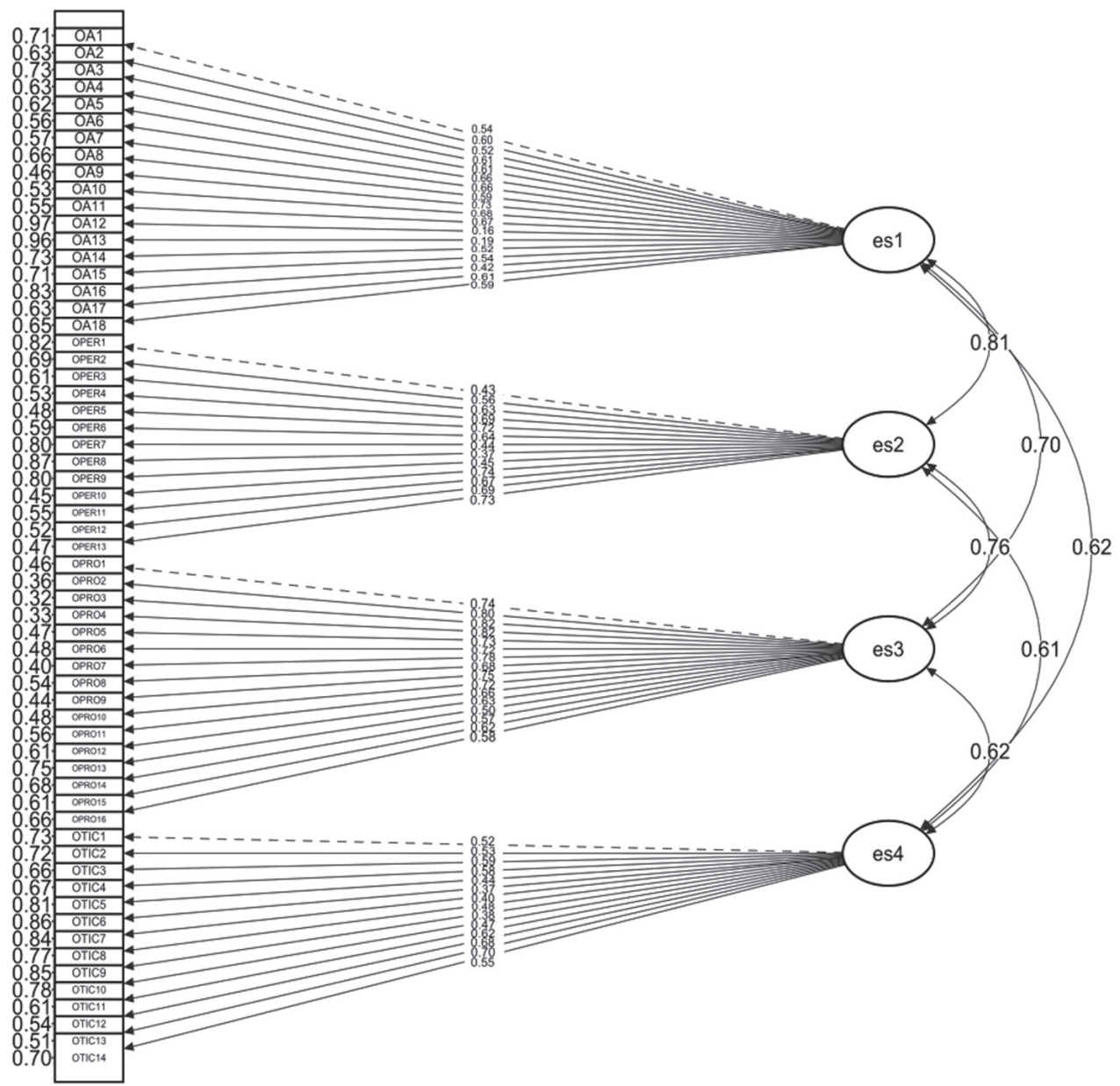

Figura 2. Estimaciones del modelo para la escala POTAE

\section{Fiabilidad del instrumento}

En nuestro caso, dada la situación en las que nos encontramos de un estudio piloto, no ha sido posible el cálculo de la fiabilidad de las puntuaciones a través de la vía test-retest, por tanto, la fiabilidad del cuestionario fue estimada a través del cálculo del coeficiente $\alpha$ (alfa de Cronbach), para determinar la consistencia interna, obteniéndose un coeficiente igual a .96 para toda la escala. En cada dimensión, la fiabilidad obtenida ha sido de .89 para Orientación Académica, .85 para Orientación Personal, .94 para Orientación Profesional y .87 para Orientación y TIC. 


\section{DISCUSIÓN}

El objetivo central de esta investigación ha sido diseñar y validar una escala para la evaluación de la tutoría universitaria con la capacidad suficiente para desarrollar un trabajo posterior de detección de necesidades y desarrollar, a partir del mismo, un Sistema de Recomendación basado en necesidades de orientación y tutoría de alumnado y egresados universitarios. Para ello se ha creado la escala POTAE, fundamentada en las tres grandes dimensiones de la orientación: académica, personal, profesional, a la que se añadió otra transversal, emergente, que proviene del uso de las TIC en la labor tutorial. Sobre las mismas, se define la práctica tutorial universitaria apoyada en un planteamiento teórico que sustenta la concreción operativa de los ítems y confiere consistencia al conjunto de la escala.

Con el propósito de procurar la validez desde más de una evidencia, este trabajo complementa la información recabada mediante el procedimiento de juicio de expertos (evidencias de validez basada en el contenido) con otras procedentes de la validación de su estructura interna o de constructo del instrumento elaborado, y la validación de contenido del mismo a través de propuestas de mejora propuestas por los expertos, con lo que se obtuvo una información de gran utilidad para depurar el instrumento y afianzar el diseño final del mismo. La mayoría de las modificaciones sugeridas por los expertos, se dirigían a la redacción y a la amplitud del cuestionario.

Posteriormente, los estudios psicométricos realizados sobre la escala muestran una sólida y robusta configuración de las dimensiones y subdimensiones que subyacen al instrumento. En todas ellas, los análisis han sido pertinentes, dados los altos índices KMO obtenidos, con valores comprendidos entre .742 y .837 , lo que permite concluir la conveniencia del análisis factorial. Asimismo, la prueba de esfericidad de Bartlett, que evalúa la aplicabilidad del análisis factorial de todas las escalas, tiene un valor de 6701.698 y una significación $p=.000$, por lo que los análisis factoriales pueden aplicarse. La medida de adecuación muestral (MSA) corrobora de alguna forma los valores anteriores, en este caso con un índice .97.

El AFC ha completado la validez de constructo, señalando una calidad métrica adecuada y un ajuste satisfactorio del modelo. Se confirma el carácter multidimensional del instrumento y la capacidad del mismo para medir las dimensiones de la tutoría universitaria, así como los diferentes aspectos de la tutoría que guardan entre sí altos índices de correlación, muy estables entre ellos. A esto se añade, el hecho de que la muestra de participantes en la validación del instrumento ha sido obtenida mediante un muestreo aleatorio proporcional, algo que es inusual en este tipo de 
estudios. Se favorece de esta forma la creación de un instrumento que puede servir para generalizar resultados sobre la tutoría universitaria.

El rigor de la fundamentación teórica ha favorecido que el instrumento tenga una alta fiabilidad global y una consistencia interna adecuada. La dimensión profesional presenta la fiabilidad más alta, y la personal la más baja (.85), lo que guarda relación con el carácter polémico de esta última -ya señalado por García-Antelo (2011)-, debido en gran medida a la falta de formación del profesorado. Por su parte, las dimensiones académica y uso de las TIC se sitúan en valores cercanos a .90. En cada dimensión la fiabilidad obtenida ha sido de .89 para Orientación Académica, .85 para Orientación Personal, .94 para Orientación Profesional y .87 para Orientación y TIC. Las cuatro grandes dimensiones, tienen un firme sustento en la literatura, tanto desde un punto de vista teórico como en investigaciones llevadas a cabo en los últimos años.

La puesta en práctica de este instrumento con otros parecidos realizados en diversas investigaciones (López-Gómez, 2015), puede aportar una importante detección de ideas previas y un estudio de partida para que cada universidad analice orientaciones sobre su buen funcionamiento, organización y gestión tanto a nivel personal, académico y profesional, además de poner en valor el grado de gestión de la misma mediante las TIC. El instrumento creado puede resultar útil y novedoso dado el enfoque eminentemente práctico que adopta el mismo, diferente de otras propuestas similares ya señaladas.

Entre las principales limitaciones de este estudio cabe destacar el hecho de que solo se ha considerado el alumnado de $2^{\circ}$ y $4^{\circ}$ cursos de distintos grados y postgrado de diferentes universidades, por lo que sería interesante aplicar la escala a todos los cursos; $y$, por otra parte, la ausencia de estudios que hayan validado escalas con un objetivo similar. De igual forma, hubiera sido deseable poder haber abarcado una mayor muestra de sujetos de universidades europeas, pero la diversidad en los planteamientos de los estudios lo ha imposibilitado.

\section{AGRADECIMIENTOS}

Este trabajo se produjo en el marco del proyecto I+D de Excelencia "TIMONEL: Sistema de Recomendación (SR) basado en necesidades de orientación y tutoría de alumnado y egresados universitarios europeos", aprobado en la convocatoria de 2016 del Ministerio de Economía y Competitividad de España (Ref. EDU2016-75892-P). 


\section{ANEXO}

\section{Cuestionario "La práctica orientadora y tutorial en alumnado y egresados universitarios (POTAE-17)"}

\begin{tabular}{|l|l|l|lr|}
\hline Datos de identificación: \\
\hline Universidad & \multicolumn{3}{l|}{} \\
\hline Facultad & \multicolumn{1}{l|}{ Sexo } & Hombre $\square \quad$ Mujer $\square$ \\
\hline $\begin{array}{l}\text { Curso que } \\
\text { estudias }\end{array}$ & $\begin{array}{l}2^{\circ} \square 4^{\circ} \square \\
\text { Máster } \square \text { Doctorado } \square\end{array}$ & Edad & & \\
\hline $\begin{array}{l}\text { ¿Participas o has participado en un Plan de Acción } \\
\text { Tutorial (PAT)? }\end{array}$ & Sí $\square \quad$ No $\square$ \\
\hline $\begin{array}{l}\text { En caso afirmativo, indica el } \\
\text { número de años }\end{array}$ & & & \\
\hline
\end{tabular}

A continuación, te pedimos que señales en cada casilla el grado de acuerdo o desacuerdo con cada ítem en función de la siguiente escala:

$1=$ Totalmente en desacuerdo 3= De acuerdo 4= Bastante de acuerdo $5=$ Totalmente de acuerdo

Muchas gracias

\begin{tabular}{|c|c|c|c|c|c|}
\hline \multicolumn{6}{|l|}{ ORIENTACIÓN ACADÉMICA } \\
\hline & 1 & 2 & 3 & 4 & 5 \\
\hline $\begin{array}{l}\text { 1. El profesorado me resuelve los problemas académi- } \\
\text { cos que tengo. }\end{array}$ & & & & & \\
\hline $\begin{array}{l}\text { 2. Se promueve el razonamiento crítico en las clases } \\
\text { para la toma de decisiones. }\end{array}$ & & & & & \\
\hline $\begin{array}{l}\text { 3. Se relacionan los aprendizajes de las diferentes asig- } \\
\text { naturas. }\end{array}$ & & & & & \\
\hline $\begin{array}{l}\text { 4. En las clases se desarrollan diferentes metodologías } \\
\text { de trabajo }\end{array}$ & & & & & \\
\hline $\begin{array}{l}\text { 5. El profesorado realiza feedback o retroalimentación } \\
\text { sobre los logros académicos. }\end{array}$ & & & & & \\
\hline $\begin{array}{l}\text { 6. Se da respuesta a las necesidades específicas de } \\
\text { apoyo educativo de los estudiantes. }\end{array}$ & & & & & \\
\hline $\begin{array}{l}\text { 7. Durante la carrera me asesoran sobre las actividades } \\
\text { de formación complementarias. }\end{array}$ & & & & & \\
\hline
\end{tabular}




\begin{tabular}{|c|c|c|c|c|c|}
\hline \multicolumn{6}{|l|}{ ORIENTACIÓN ACADÉMICA } \\
\hline & 1 & 2 & 3 & 4 & 5 \\
\hline $\begin{array}{l}\text { 8. Me orientan sobre el desarrollo de los TFG, TFM, } \\
\text { Doctorado, etc. }\end{array}$ & & & & & \\
\hline $\begin{array}{l}\text { 9. Se promueve la motivación del alumnado hacia el } \\
\text { aprendizaje. }\end{array}$ & & & & & \\
\hline $\begin{array}{l}\text { 10. Se utilizan técnicas de estudio en las diferentes asig- } \\
\text { naturas. }\end{array}$ & & & & & \\
\hline $\begin{array}{l}\text { 11. El profesorado facilita ayuda en el diseño del itinera- } \\
\text { rio académico (elección de optativas, especialidades, } \\
\text { menciones, etc.) }\end{array}$ & & & & & \\
\hline $\begin{array}{l}\text { 12. A lo largo de la carrera he asistido tutorías de forma } \\
\text { individual. }\end{array}$ & & & & & \\
\hline $\begin{array}{l}\text { 13. A lo largo de la carrera he asistido tutorías de forma } \\
\text { grupal. }\end{array}$ & & & & & \\
\hline $\begin{array}{l}\text { 14. Me han informado sobre la normativa que regula la } \\
\text { acción tutorial en mi universidad. }\end{array}$ & & & & & \\
\hline $\begin{array}{l}\text { 15. La universidad da a conocer los programas existen- } \\
\text { tes para la ampliación de estudios, doble titulación, } \\
\text { movilidad nacional e internacional, etc. }\end{array}$ & & & & & \\
\hline $\begin{array}{l}\text { 16. Me han explicado las guías docentes de las diferen- } \\
\text { tes asignaturas. }\end{array}$ & & & & & \\
\hline $\begin{array}{l}\text { 17. Me han orientado sobre cómo afrontar los exáme- } \\
\text { nes. }\end{array}$ & & & & & \\
\hline $\begin{array}{l}\text { 18. Me han asesorado sobre la realización de los traba- } \\
\text { jos }\end{array}$ & & & & & \\
\hline $\begin{array}{l}\text { 19. ¿Qué puntuación global otorgas a la orientación } \\
\text { académica recibida hasta ahora? (De } 0 \text { a 10) }\end{array}$ & & & & & \\
\hline
\end{tabular}

\begin{tabular}{|l|l|l|l|l|l|}
\hline \multicolumn{4}{|c|}{ ORIENTACIÓN PERSONAL } & & \\
\hline $\begin{array}{l}\text { 1. En las clases se fomenta la tolerancia y respeto mu- } \\
\text { tuo en el grupo. }\end{array}$ & & & & & \\
\hline $\begin{array}{l}\text { 2. El profesorado nos orienta personalmente cuando lo } \\
\text { necesitamos. }\end{array}$ & & & & & \\
\hline $\begin{array}{l}\text { 3. Tenemos ayuda del profesorado para resolver con- } \\
\text { flictos en el aula. }\end{array}$ & & & & & \\
\hline $\begin{array}{l}\text { 4. Conozco los servicios que ofrece la universidad para } \\
\text { el apoyo y orientación a nivel personal y/o social. }\end{array}$ & & & & & \\
\hline
\end{tabular}




\begin{tabular}{|c|c|c|c|c|c|}
\hline \multicolumn{6}{|l|}{ ORIENTACIÓN PERSONAL } \\
\hline & 1 & 2 & 3 & 4 & 5 \\
\hline $\begin{array}{l}\text { 5. Me asesoran sobre los servicios especializados exis- } \\
\text { tentes (pedagógicos, psicológicos, etc.) }\end{array}$ & & & & & \\
\hline $\begin{array}{l}\text { 6. Me han informado sobre cómo actuar ante cualquier } \\
\text { situación de acoso. }\end{array}$ & & & & & \\
\hline $\begin{array}{l}\text { 7. En la universidad se fomenta la igualdad por razón } \\
\text { de género. }\end{array}$ & & & & & \\
\hline 8. Existe un clima de respeto a la diversidad en el aula. & & & & & \\
\hline $\begin{array}{l}\text { 9. Participo en actividades universitarias complemen- } \\
\text { tarias. }\end{array}$ & & & & & \\
\hline 10. Me orientan en el autoconocimiento personal. & & & & & \\
\hline $\begin{array}{l}\text { 11. Durante la carrera se facilita el desarrollo de habili- } \\
\text { dades sociales. }\end{array}$ & & & & & \\
\hline $\begin{array}{l}\text { 12. A lo largo de los estudios se fomenta el desarrollo de } \\
\text { hábitos saludables. }\end{array}$ & & & & & \\
\hline $\begin{array}{l}\text { 13. Se desarrollan competencias emocionales en las dis- } \\
\text { tintas materias. }\end{array}$ & & & & & \\
\hline $\begin{array}{l}\text { 14. ¿Qué puntuación global otorgas a la orientación } \\
\text { personal recibida hasta ahora? (De } 0 \text { a 10) }\end{array}$ & & & & & \\
\hline
\end{tabular}

\begin{tabular}{|c|c|c|c|c|c|}
\hline \multicolumn{6}{|l|}{ ORIENTACIÓN PROFESIONAL } \\
\hline & 1 & 2 & 3 & 4 & 5 \\
\hline $\begin{array}{l}\text { 1. Los estudiantes estamos informados sobre la transi- } \\
\text { ción al mundo laboral. }\end{array}$ & & & & & \\
\hline $\begin{array}{l}\text { 2. Me ayudan en el proceso de toma de decisiones vo- } \\
\text { cacionales. }\end{array}$ & & & & & \\
\hline $\begin{array}{l}\text { 3. Me orientan en el diseño de mi itinerario profesio- } \\
\text { nal. }\end{array}$ & & & & & \\
\hline $\begin{array}{l}\text { 4. Recibo asesoramiento sobre competencias para la } \\
\text { inserción laboral. }\end{array}$ & & & & & \\
\hline $\begin{array}{l}\text { 5. Recibo orientación en la elaboración de mi currícu- } \\
\text { lum vitae. }\end{array}$ & & & & & \\
\hline $\begin{array}{l}\text { 6. Estoy informado sobre las posibilidades de empren- } \\
\text { dimiento en la titulación. }\end{array}$ & & & & & \\
\hline $\begin{array}{l}\text { 7. He sido orientado sobre nuevos yacimientos de em- } \\
\text { pleo. }\end{array}$ & & & & & \\
\hline $\begin{array}{l}\text { 8. He recibido pautas sobre cómo realizar una entre- } \\
\text { vista de trabajo. }\end{array}$ & & & & & \\
\hline
\end{tabular}




\begin{tabular}{|c|c|c|c|c|c|}
\hline \multicolumn{6}{|l|}{ ORIENTACIÓN PROFESIONAL } \\
\hline & 1 & 2 & 3 & 4 & 5 \\
\hline $\begin{array}{l}\text { 9. Me han facilitado ayuda para crear redes profesiona- } \\
\text { les. }\end{array}$ & & & & & \\
\hline $\begin{array}{l}\text { 10. Me han informado sobre ofertas de empleo público } \\
\text { y/o privado. }\end{array}$ & & & & & \\
\hline $\begin{array}{l}\text { 11. Recibo orientación sobre prácticas de empresas en } \\
\text { colaboración con la universidad. }\end{array}$ & & & & & \\
\hline $\begin{array}{l}\text { 12. He sido informado sobre la oferta de prácticas profe- } \\
\text { sionales en el extranjero. }\end{array}$ & & & & & \\
\hline $\begin{array}{l}\text { 13. Conozco las expectativas profesionales de mi titula- } \\
\text { ción. }\end{array}$ & & & & & \\
\hline $\begin{array}{l}\text { 14. Relaciono los contenidos de las asignaturas con sus } \\
\text { salidas profesionales. }\end{array}$ & & & & & \\
\hline $\begin{array}{l}\text { 15. Desarrollo competencias profesionales mediante si- } \\
\text { mulaciones de casos reales. }\end{array}$ & & & & & \\
\hline $\begin{array}{l}\text { 16. Mantengo contactos profesionales con empresas y/o } \\
\text { instituciones relacionadas con la titulación. }\end{array}$ & & & & & \\
\hline $\begin{array}{l}\text { 17. ¿Qué puntuación global otorgas a la orientación } \\
\text { profesional recibida hasta ahora? (De } 0 \text { a 10) }\end{array}$ & & & & & \\
\hline
\end{tabular}

\section{ORIENTACIÓN Y TIC}

\section{$1=$ Totalmente en desacuerdo $2=$ Bastante en desacuerdo $3=\mathrm{De}$} acuerdo 4= Bastante de acuerdo $5=$ Totalmente de acuerdo

\begin{tabular}{|l|l|l|l|l|l|}
\hline & 1 & 2 & 3 & 4 & 5 \\
\hline 1. En las clases se fomenta el dominio de las TIC. & & & & & \\
\hline 2. Conozco la búsqueda de trabajo por internet. & & & & & \\
\hline $\begin{array}{l}\text { 3. El profesorado, en general, dispone de página Web } \\
\text { profesional. }\end{array}$ & & & & \\
\hline 4. La página Web del profesorado está actualizada. & & & & & \\
\hline $\begin{array}{l}\text { 5. En la plataforma de la universidad se encuentra in- } \\
\text { formación relacionada con mis asignaturas. }\end{array}$ & & & & & \\
\hline 6. En tutoría utilizo el correo electrónico. & & & & & \\
\hline $\begin{array}{l}\text { 7. Utilizo la videoconferencia (Skype o similar) en mis } \\
\text { tutorías. }\end{array}$ & & & & & \\
\hline $\begin{array}{l}\text { 8. En general, el profesorado dispone de una red social } \\
\text { (Facebook o similar) con sus alumnos tutelados. }\end{array}$ & & & & & \\
\hline $\begin{array}{l}\text { 9. El grupo de clase tiene un grupo de WhatsApp en el } \\
\text { que participa el profesorado. }\end{array}$ & & & & & \\
\hline
\end{tabular}




\begin{tabular}{|c|c|c|c|c|c|}
\hline \multicolumn{6}{|l|}{ ORIENTACIÓN Y TIC } \\
\hline \multicolumn{6}{|c|}{$\begin{array}{l}\text { 1= Totalmente en desacuerdo } 2=\text { Bastante en desacuerdo } 3=\text { De } \\
\text { acuerdo } 4=\text { Bastante de acuerdo } 5=\text { Totalmente de acuerdo }\end{array}$} \\
\hline & 1 & 2 & 3 & 4 & 5 \\
\hline $\begin{array}{l}\text { 10. Dispongo de un foro específico en la plataforma de } \\
\text { la universidad. }\end{array}$ & & & & & \\
\hline $\begin{array}{l}\text { 11. Existe un repositorio de recursos digitales a nuestra } \\
\text { disposición. }\end{array}$ & & & & & \\
\hline $\begin{array}{l}\text { 12. Dispongo de un listado de enlaces a páginas Web } \\
\text { que me sirvan de ayuda como orientación en las } \\
\text { asignaturas. }\end{array}$ & & & & & \\
\hline $\begin{array}{l}\text { 13. Conozco recursos o redes digitales sobre mis estu- } \\
\text { dios. }\end{array}$ & & & & & \\
\hline $\begin{array}{l}\text { 14. Estoy informado de las posibilidades que tiene el te- } \\
\text { letrabajo. }\end{array}$ & & & & & \\
\hline $\begin{array}{l}\text { 15. ¿Qué puntuación global otorgas al uso que has reali- } \\
\text { zado de las TIC en la orientación educativa recibida? } \\
\text { (De } 0 \text { a 10) }\end{array}$ & & & & & \\
\hline
\end{tabular}

A continuación, dispones de un espacio para expresar tu opinión personal sobre los diferentes aspectos recogidos en este cuestionario y otros que te parezcan que pueden ser de interés. 


\section{REFERENCIAS BIBLIOGRÁFICAS}

Álvarez-Pérez, P.R. (2013). La tutoría como eje articulador del proceso de aprendizaje del alumnado. Qurriculum: Revista de Teoría, Investigación y Práctica Educativa 26, 73-87.

Amor, M.I., y Dios, I. (2017). La tutoría universitaria: un espacio para la orientación personal, académica y profesional en la formación inicial del profesorado. REOP-Revista Española de Orientación y Psicopedagogía, 28(1), 119-130.

Arraiz, A., Berbegal, A., y Sabiron, F. (2018). La tutoría académica focalizada en la evaluación: análisis de necesidades desde la perspectiva de estudiantes y profesores. REDU: Revista de Docencia Universitaria, 16(2), 211-229.

Byrne, B.M. (2006). Structural equation modeling with AMOS. Basic Concepts, Applications, and Programming. Taylor and Francis.

Caldera, J.F., Carranza, M.R., Jiménez, A.A., y Pérez, I. (2015). Actitudes de los estudiantes universitarios ante la tutoría. Diseño de una escala de medición. Revista de la Educación Superior, 44(173), 103-124.

Cambours, A.M., Iglesias, A.I., y Muiños, S.M. (2017). La tutoría en la Universidad: una estrategia para la retención de los estudiantes. https://bit. ly/2wem2sR

Campos, A.C., y Oliveira, A.H. (2013). A identidade do professor Tutor. Revista ADAPECI, Educação a Distância e Práticas Educativas Comunicacionais e Inter-culturais, 13(1), 23-37.

Cano, R. (2009). Tutoría universitaria y aprendizaje por competencias.
¿Cómo lograrlo? Revista Electrónica Interuniversitaria de Formación del Profesorado- REIFOP, 12(1), 181-204.

Capelari, M. (2014). Las Políticas de Tutoría en la Educación Superior: Génesis, Trayectorias e Impactos en Argentina y México. Revista Latinoamericana de Educación Comparada, 5(5), 41-54.

Castellano, E.A., y Pantoja, A. (2017). Eficacia de un programa de intervención basado en el uso de las TIC en la tutoría. Revista de Investigación Educativa, 35(1), 215233.

Clerici, R., y Da Re, L. (2019). Evaluación de la eficacia de un programa de tutoría formativa. Revista de investigación educativa, 37(1), 39-56.

Coertjens, L., Donche, V., De Maeyer, S., van Daal, T., \& Van Petegem, P. (2017). The growth trend in learning strategies during the transition from secondary to higher education in Flanders. Higher Education, 73(3), 499-518.

De la Cruz, G. (2017). Tutoría en Educación Superior: análisis desde diferentes corrientes psicológicas e implicaciones prácticas. $C P U-e$, Revista de Investigación Educativa, 25, 34-59.

European Lifelong Guidance Policy Network (2015). European Lifelong Guidance Policies: Summative Report 2007-15. A report on the work of the European Lifelong Guidance Policy Network 2007-15. ELGPN.

Farr, B., Charles, M.B., Keast, R., Woolcott, G., y Chamberlain, D. (2018). Por qué los profesores siguen siendo importantes: el impacto 
del intercambio entre profesores y estudiantes sobre el compromiso de los estudiantes y la intención de abandonar la universidad prematuramente. Educación Superior, 75(1), 167-185.

Feldt, R.C, Graham, M., \& Dew, D. (2011). Measuring Adjustment to College: Construct Validity of the Student Adaptation to College Questionnaire. Measurement and Evaluation in Counseling and Development, 44(2), 92-104.

Fernández, B., y Cabero, J. (2016). Percepciones de teleformadores del Instituto de Formación y Estudios Sociales de Sevilla sobre la teleformación. Innoeduca. International Journal of Technology and Educational Innovation, 2(1), 4-12.

Fernández, M.D., y Sanjuán, M.M. (2014). ¿Qué uso hacen del e-learning nuestros estudiantes? Píxel-Bit. Revista de Medios y Educación, 45, 137-156.

Fernández, M.A., Mena, E., y Tójar, J.C. (2017). Funciones de la tutoría en e-learning: Estudio mixto de los roles del tutor online. Revista de Investigación Educativa, 35(2), 409-426. https://doi.org/10.6018/ rie.35.2.273271

García-Antelo, B. (2011). La tutoría en la universidad: percepción de alumnado $y$ profesorado. ICE Universidad de Santiago de Compostela.

Gaviria, J.L. (2000). Cambios en las técnicas cuantitativas de investigación socio-educativa. En Sociedad Española de Pedagogía (Ed.), Actas del XII Congreso Nacional y I Iberoamericano de Pedagogía (pp. 39-45). S.E.P.

Gil, J.A. (2003). Métodos de investigación en educación. Análisis discriminante. UNED.
Holliman, A.J., Martin, A.J., \& Collie, R.J. (2018). Adaptability, engagement, and degree completion: a longitudinal investigation of university students. Educational Psychology, 38(6), 785799.

Larose, S., Cyrenne, D., Garceau, O., Harvey, M., Guay, F., Godin, F., \& Deschênes, C. (2011). Academic mentoring and dropout prevention for students in math, science and technology. Mentoring y Tutoring: Partnership in Learning, 19(4), 419439.

León, V., y Lugo, E. (2008). Los programas institucionales de tutoría en la universidad pública en México: El caso de las universidades públicas estatales de la región Centro-Sur de la ANUIES. Universidad Autónoma del Estado de Morelos.

Liu, O. L., y Rijmen, F. (2008). A modified procedure for parallel analysis of ordered categorical data. Behavior Research Methods, 40, 556562.

López-Gómez, E. (2015). La tutoría en el EEES: propuesta, validación y valoración de un modelo integral. [Tesis Doctoral]. UNED.

Lorenzo, G., Carreres, A.L., Lledó, A. L., y Vera, G. A. (2017). La acción tutorial como acompañamiento en el alumnado universitario con discapacidad: hacia una educación inclusiva. International Journal of Developmental and Educational Psychology. Revista INFAD de Psicología, 4(1), 137-144.

Magen, N., \& Shachar, H. (2017). Quality of teaching and dropout risk: Amultilevel analysis. Journal of Education for Students Placed at Risk, 22(1), 9-24. https://doi.org/7710.1080/11356405.20 15.1124548 
Michavila, F., y García, J. (2003). La tutoría y los nuevos modos de aprendizaje en la universidad. Cátedra UNESCO de Gestión y Política Universitaria.

Morales, F., Urosa, B., y Blanco, A. (2000). Construcción de escalas de actitudes tipo Likert. La Muralla.

Muñoz, J.L., y Gairin, J. (2016). Orientación y tutoría durante los estudios universitarios: el plan de acción tutorial. Revista Fuentes, 14 172-192.

Pantoja, A. (2009). La acción tutorial ante el reto de la Tecnologías de la Información y la Comunicación (TIC). Wolters Kluwer.

Respondek, L., Seufert, T., Stupnisky, R., \& Nett, U.E. (2017). Perceived academic control and academic emotions predict undergraduate university student success: Examining effects on dropout intention and achievement. Frontiers in Psychology, 8, 243, 1-18.

Rodríguez, S. (2004). Manual de tutoría universitaria. Octaedro.

Rodríguez, C., y Calvo, A. (2011). La figura del tutor de e-learning. Aportaciones de una investigación con estudios de caso. Universities and Knowledge Society Journal, 8(1), 66-77.

Sánchez, P.P. López, M.M., y Cienfuegos, Y.A. (2018). La orientación educativa en la actividad pedagógica profesional del docente universitario. Revista Conrado, 14(65), 50-57.
Siegel, S., y Castellan, N.J. (1995). Estadística no paramétrica aplicada a las ciencias de la conducta. Trillas.

Steiger, J.H., \& Lind, J.C. (1980). Statistically-based tests for the number of common factors. Paper presented at the Annual Spring Meeting of the Psychometric Society. Iowa City, IA.

Tinto, V. (2017). Through the eyes of students. Journal of College Student Retention: Research, Theory $y$ Practice, 19(3), 254-269.

Van, E.C., Jansen, E.P., \&Van de Grift, W.J. (2017). First-year university students' academic success: the importance of academic adjustment. European Journal of Psychology of Education, 33, 749-767. https://doi. org/10.1007 / s10212-017-0347-8.

Venegas, L., y Gairín, J. (2018). Gestión y desarrollo de planes de acción tutorial en la universidad. Estudios de casos. Revista Española de Orientación y Psicopedagogía, 29(1), 125-143.

Watts, A.G. (1990). The Role of Guidance in Educational Change. En A.G. Watts (edit.), Guidance and educational change: a cross - sectorial review of policy and practice (pp. 10-13). CRAC Pub., Hobsons Pub.

Weng, L.J., \& Cheng, Ch.P. (2005) Parallel Analysis with Unidimensional Binary Data. Educational and Psychological Measurement, 65, 697716.

Wisker, G., Exley, K., Antoniou, M., \& Ridley, P. (2013). Working one-to-one with students: Supervising, coaching, mentoring, and personal tutoring. Routledge. 


\section{PERFIL ACADÉMICO Y PROFESIONAL DE LOS AUTORES}

Antonio Pantoja-Vallejo. ORCID: http://orcid.org/0000-0001-5374-4378

Profesor Titular de Universidad. Responsable del Grupo de Investigación IDEO (HUM 660) e Investigador Principal del Proyecto I+D de Excelencia TIMONEL (Ref. EDU2016-75892-P). Editor jefe de las revistas científicas REID y MLSER. Líneas de investigación: orientación educativa, tutoría, uso de las TIC en las mismas. Email: apantoja@ujaen.es

David Molero. ORCID: http://orcid.org/0000-0002-0392-4351

Profesor Titular de Universidad. Miembro del Grupo IDEO HUM-660 "Investigación y Desarrollo Educativo de la Orientación" de la Universidad de Jaén. Línea de investigación: educación emocional, orientación y evaluación educativa. Email: dmolero@ujaen.es

M. Dolores Molina-Jaén. ORCID: http://orcid.org/0000-0001-6010-6382

Profesora Catedrática del CU. Miembro del Grupo IDEO HUM-660 "Investigación y Desarrollo Educativo de la Orientación" de la Universidad de Jaén. Líneas de investigación: desarrollo de las TIC en la metodología y escuela inclusiva. Email: mjruiz@ujaen.es

M. Jesús Colmenero-Ruiz. ORCID: http://orcid.org/0000-0003-35592512

Profesora Titular de Universidad. Miembro del Grupo de investigación IDEO (HUM-660) "Investigación y Desarrollo Educativo de la Orientación". Editora de la revista científica MLSER. Líneas de investigación: inclusión educativa, orientación y formación del profesorado. Email: mdmolina@ fundacionsafa.es

Fecha Recepción del Artículo: 24. Septiembre. 2019

Fecha Modificación del Artículo: 17. Diciembre. 2019

Fecha Aceptación del Artículo: 18. Diciembre. 2019

Fecha Revisión para Publicación: 20. Enero. 2020 